\title{
Cavity ringdown laser absorption spectroscopy and time-of-flight mass spectroscopy of jet-cooled silver silicides
}

\author{
J. J. Scherer, J. B. Paul, C. P. Collier, and R. J. Saykally \\ Department of Chemistry, University of California, Berkeley, California 94720-1460
}

(Received 15 February 1995; accepted 27 March 1995)

\begin{abstract}
The cavity ringdown technique has been employed for the first spectroscopic characterization of the $\mathrm{AgSi}$ molecule, which is generated in a pulsed laser vaporization plasma reactor. A total of 20 rovibronic bands between 365 and $385 \mathrm{~nm}$ have been measured and analyzed to yield molecular properties for the $X, B$, and $C^{2} \Sigma$ states of $\mathrm{AgSi}$. A time-of-flight mass spectrometer simultaneously monitors species produced in the molecular beam and has provided the first direct evidence for the existence of polyatomic silver silicides. Comparison of the AgSi data to our recent results for the $\mathrm{CuSi}$ diatom reveals very similar chemical bonding in the two coinage metal silicides, apparently dominated by covalent interactions. (C) 1995 American Institute of Physics.
\end{abstract}

\section{INTRODUCTION}

In the last two decades there has been an enhanced interest in the formation mechanisms and properties of metal silicides at metal-silicon interfaces. This interest is primarily motivated by the associated consequences of silicide formation at the metal-silicon interface for semiconductor and microelectronics devices. Numerous solid state experimental techniques have been implemented to detect metal silicides and determine their respective properties, such as (Schottky) barrier heights and contact resistances. ${ }^{1-3}$ In particular, the coinage metal silicides have received much attention, due to their common use in electronic devices. Several studies have focused on the trends observed for silicide formation in $\mathrm{Cu}$, $\mathrm{Ag}$, and $\mathrm{Au}$ films deposited on silicon substrates.

The formation mechanisms and associated properties of silver silicides in particular have been the focus of much solid state experimental research. As with copper silicides, the goals of this research include deducing electrical properties of silver-silicon junctions, ${ }^{4}$ studying the glass forming properties of $\mathrm{Ag}-\mathrm{Si}$ alloys, ${ }^{5,6}$ and characterizing $\mathrm{Ag}-\mathrm{Si}$ surface structures. ${ }^{7}$ In the work of Arnold et al., metallurgically grown Schottky junctions were produced in directionally solidified eutectic $\mathrm{Ag}-\mathrm{Si}$ alloys. ${ }^{8}$ From the current-voltage ( $I$ $V)$ measurements made in that study, the crystallites were found to be $n$ doped and several current flow mechanisms were postulated to exist, although the specific nature of these mechanisms was not addressed. Diffusion of silver into silicon lattices has been evidenced in several studies, although the presence of ordered phases, as is found in the case of copper silicides, has not yet been established. ${ }^{9}$ In another study, it was found that $\mathrm{Au}$ and $\mathrm{Cu}$ silicides were readily formed at room temperature, while heating to $400{ }^{\circ} \mathrm{C}$ was required for Ag silicide formation. ${ }^{10}$ Similarly, peculiarities of $\mathrm{Ag}$ silicides formed at $\mathrm{Ag}-\mathrm{Si}$ interfaces have been observed, suggesting that random phases dominate the interface composition. $^{11}$

In spite of the large body of solid state work, the formation mechanisms and properties of metal silicides are still not well characterized. Moreover, only a few gas phase studies of metal silicides and $a b$ initio results exist. In the case of silver silicides, the only previous gas phase work consists of measurement of the dissociation energy of the $\mathrm{AgSi}$ diatom, deduced in the mass spectrometric studies of Riekert et al. ${ }^{12}$ In an effort to elucidate the properties of molecular metal silicides, we have employed cavity ringdown laser absorption spectroscopy (CRLAS) and time-of-flight mass spectroscopy (TOFMS) for the study of jet-cooled metal silicides. Our initial efforts have focused on the coinage metal silicides, $\mathrm{Cu}_{x} \mathrm{Si}_{y}$ and $\mathrm{Ag}_{x} \mathrm{Si}_{y}$. The results for $\mathrm{CuSi}$ have been presented in a recent publication. ${ }^{13}$ In this paper, we present the results obtained for silver silicides, which include the first gas phase spectra for any silver silicide as well as the first direct evidence for the existence of polyatomic silver silicides.

\section{EXPERIMENT}

\section{A. Cavity ringdown laser absorption spectroscopy (CRLAS)}

In the pulsed cavity ringdown technique, first developed by O'Keefe and Deacon in 1987 for the determination of mirror reflectivities, ${ }^{14}$ molecular absorption is deconvoluted from the measured photon decay time of a high finesse optical cavity which is injected with a short pulse of laser light. The high sensitivity of this technique was first demonstrated by measuring doubly forbidden transitions in molecular oxygen. Since our first application of the cavity ringdown technique to the study of pulsed molecular beams, ${ }^{15}$ several other groups ${ }^{16}$ have employed the technique for various purposes, including adaptation to kinetics studies, ${ }^{17}$ the spectroscopy of $\mathrm{OH}$ in flames, ${ }^{18}$ and the detection of $\mathrm{CH}_{3}$ in a pyrolysis cell. ${ }^{19}$

The Berkeley cavity ringdown laser absorption spectrometer and time-of-flight mass spectrometer (TOFMS) are diagrammed in Fig. 1. The apparatus has been described in detail elsewhere ${ }^{13}$ and will therefore only be briefly outlined here. The design incorporates three stages of differential pumping, consisting of a main source chamber evacuated by a large Roots pump (2500 cfm Edwards EH4200), and two diffusion pumped regions. Each of the three regions are separated by electroformed molecular beam skimmers each having a $2 \mathrm{~mm}$ diameter (Beam Dynamics). The tandem design of the apparatus allows characterization of the molecular 


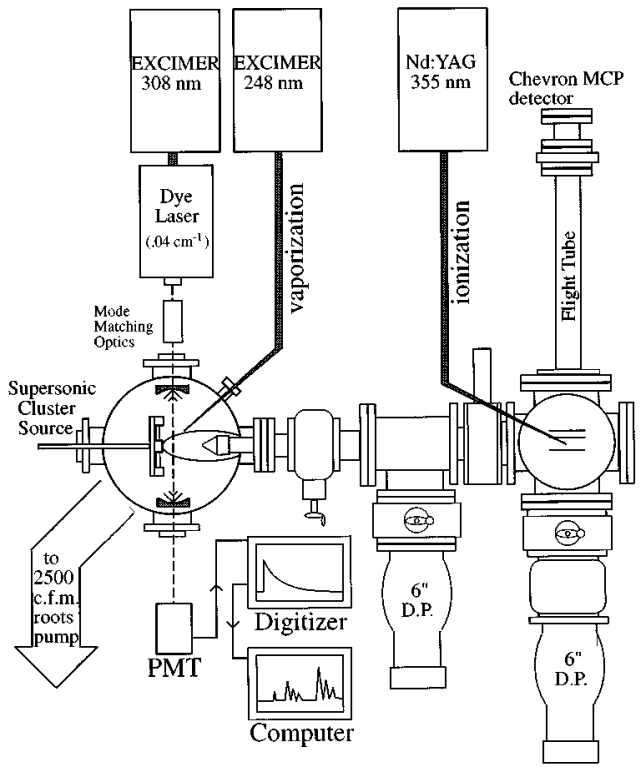

FIG. 1. Berkeley CRLAS/TOFMS apparatus: A laser vaporized pulsed molecular beam of metal clusters is generated in a vacuum chamber and perpendicularly intersected by the laser pulse train. The ringdown decay is timed to coincide with the transient molecular beam and is monitored with a photomultiplier.

beam source while simultaneously measuring absorption features, which greatly aids in the maximization of cluster concentrations and also allows correlation studies to be performed.

The ringdown laser pulse consists of a few $\mathrm{mJ}$ of narrowband $\left(0.04 \mathrm{~cm}^{-1}\right)$ excimer pumped dye laser light which is coupled into the cavity with the use of a telescope. Roughly 1 part in $10^{8}$ of this light passes through the exit mirror of the cavity and is detected with a photomultiplier (Hammamatsu R912). Proper timing of the ringdown laser pulse with respect to the transient molecular beam assures maximum temporal overlap between the two events. In fact, one of the primary advantages of employing CRLAS for pulsed molecular beams is the similar time scales of both the molecular beam and ringdown events (5-25 $\mu$ s), allowing a probe duty cycle near unity to be achieved. The resultant ringdown decay wave form is amplified, digitized with a 12 bit, $20 \mathrm{MHz}$ transient digitizer, and sent to a computer for analysis. Because the amount of light which transmits the exit mirror is linearly proportional to the amount of light still trapped inside the cavity, the intensity decay monitored at the photomultiplier follows the expression

$$
I(t)=I_{0} e^{(-T t 2 L / c)},
$$

where $T$ is the transmission coefficient of the two mirror cavity, $L$ is the mirror spacing, and $t$ is time. Measurement of the cavity "ringdown" time $\left(t\right.$, wherein $\left.I=I_{0} e^{-1}\right)$ allows the transmissivity of (or absorption inside) the cavity to be determined, if the mirror spacing (cavity length) is known. This cavity "transmissivity" represents the total losses experienced by the light pulse while traversing the cavity, including mirror transmissivity, optical scattering, mirror coating absorption, and molecular absorption for species located between the two mirrors. Measurement of the cavity "ring- down time" at a given wavelength gives a direct measurement of the total losses experienced by the circulating light over a single cavity round trip pass. A plot of cavity losses vs wavelength allows an absorption spectrum to be obtained for species placed in the cavity. Accurate relative absorption intensities for molecular transitions (in the limit of weak absorption) are readily determined by comparing cavity losses with and without the sample present. Typically, 16 laser shots are averaged per wavelength step in order to reduce noise introduced by fluctuations in the pulsed molecular beam.

\section{B. Silver silicide generation and TOFMS}

Silver silicides are produced in a pulsed UV laser vaporization plasma reactor. The apparatus has also been previously described. ${ }^{13}$ Briefly, helium carrier gas (80 psi) which contains trace amounts of silane is passed over a continuously rotating and translating silver rod which is vaporized with $\sim 200 \mathrm{~mJ}$ of tightly focused excimer laser light. The resultant laser generated plasma effectively strips the silane of its hydrogen atoms, leaving bare silicon atoms which subsequently react with the bare metal atoms to form silver silicides. As is the case with copper, the process produces bare metal clusters, silicon clusters, and metal silicides. The resultant species then rapidly cool as they expand into the evacuated chamber. Typical rotational temperatures achieved in these studies are $\sim 50 \mathrm{~K}$, substantially hotter than those typically obtained for bare metal clusters produced under the same source conditions. Removal of the silane from the carrier gas mixture returns the silver plasma from dim white to its characteristic bright green color. In fact, visual inspection of the plasma is generally a sufficient test for assuring proper source conditions for optimal silicide formation. Nonetheless, mass spectrometric measurements were performed to assure silicide production. In Fig. 2 the mass spectra of $\mathrm{Ag}_{x} \mathrm{Si}_{y}$ species are shown. In Fig. 2(a), $355 \mathrm{~nm} \mathrm{Nd:Yag} \mathrm{laser}$ light is used for photoionization of the neutral clusters in the molecular beam, while Fig. 2(b) shows the spectrum obtained when $248 \mathrm{~nm}$ excimer light is used for photoionization, under identical source conditions. Since the ionization potential (IP) for the $\mathrm{Si}$ atom is $8.151 \mathrm{eV}$ and that of $\mathrm{Ag}$ atom is $7.57 \mathrm{eV}$, the IP for the $\mathrm{Ag}_{x} \mathrm{Si}_{y}$ species is likely to lie somewhere between these two values (the IPs have not yet been experimentally determined or theoretically predicted). If this is the case, the difference between the 355 and $248 \mathrm{~nm}$ photoionization process is that in the former case three photons are likely required to ionize the neutral clusters, while in the latter, only two photons are required. The resultant difference between the two mass spectra is therefore a convolution of the two vs three photon cross sections and possible fragmentation processes which may be occurring. The similarity of the two spectra suggests that photofragmentation does not severely distort the relative intensities. In fact, the main difference between the two spectra is likely due to the higher fluence of the $355 \mathrm{~nm}$ light, which subsequently leads to more intense ion signal.

These mass spectra provide the first direct evidence for the existence of polyatomic molecular silver silicide species. The facile formation of these species may have important 

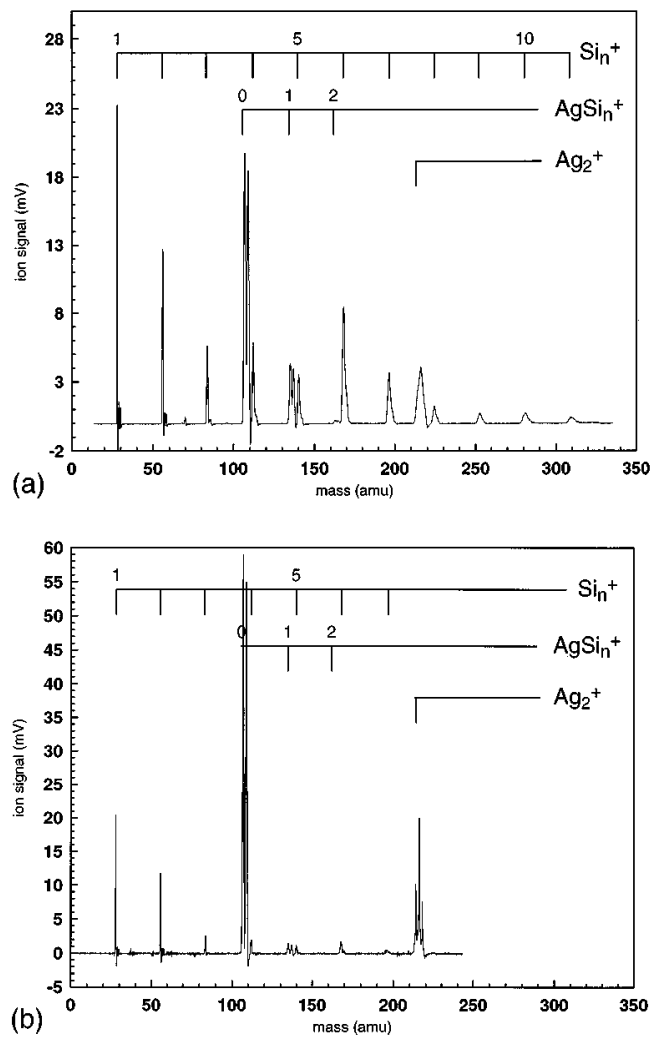

FIG. 2. TOFMS of jet-cooled silver silicides formed in the laser vaporization plasma reactor, at two different photoionization wavelengths, $355 \mathrm{~nm}$ (a) and $248 \mathrm{~nm}$ (b), under identical source conditions. The similarity of the two spectra suggests that photofragmentation does not distort the relative intensities.

implications for the possible phases present in bulk phase silver-silicon interfaces. It is notable that in contrast to the results for copper silicides, the ratio of silver silicide to bare silicon and metal cluster production is not very sensitive to the silane concentration of the carrier gas. This effect could be due to the limited range of concentrations used in these studies (typically $0.2 \%-2 \%$ ) and will require further experiments to deduce whether or not this point has any important physical significance.

\section{MOLECULAR ORBITAL CONSIDERATIONS}

There is currently no theoretical work on molecular silver silicide clusters in the literature. Therefore this discussion will be limited to simple considerations for the possible ground state symmetries for the $\mathrm{AgSi}$ molecule. The most likely candidate for the atomic asymptote for the $\mathrm{Ag}$ atom is the ${ }^{2} S$ atomic ground state, derived from the $4 d^{10} 5 s^{1}$ electronic configuration, since $\mathrm{Ag}$ has been found to readily form molecular bonds in this state (e.g., $\mathrm{Ag}_{2},{ }^{20} \mathrm{AgAu},{ }^{21} \mathrm{CuAg}$ ). ${ }^{22}$ For the silicon atom, the $3 s^{2} 3 p^{2}$ electronic configuration leads to states of ${ }^{3} P$ (ground state), ${ }^{1} D$, and ${ }^{1} S$ symmetry, in order of increasing energy. Possible ground state candidates for the AgSi diatom are therefore found by combining the ${ }^{2} S$ state of Ag with the ${ }^{3} P,{ }^{1} D$, and ${ }^{1} S$ states of Si. States resulting from the combination of ${ }^{2} S$ and ${ }^{3} P$ terms are of ${ }^{2} \Sigma$, ${ }^{2} \Pi$, ${ }^{4} \Sigma$, and ${ }^{4} \Pi$ symmetry. ${ }^{24}$ Similarly, possible states resulting from the ${ }^{2} S$ and ${ }^{1} D$ combination possess ${ }^{2} \Sigma$, ${ }^{2} \Pi$, or ${ }^{2} \Delta$ symmetry, while those resulting from the addition of ${ }^{2} S$ and ${ }^{1} S$ atomic states produce only a ${ }^{2} \Sigma$ state. From these considerations, the most likely ground state candidate for $\mathrm{AgSi}$ is one of ${ }^{2} \Sigma$ symmetry. Our current experimental results for $\mathrm{AgSi}$ and recent results for $\mathrm{CuSi}^{13}$ indicate that this is indeed the case.

The fact that our spectra are consistent with ${ }^{2} \Sigma$ ground states for both copper and silver silicides deserves some consideration. If ground state bonding in $\mathrm{AgSi}$ is due to the combination of the ${ }^{2} S \mathrm{Ag}$ atom with the ${ }^{3} \mathrm{P}$ Si atom, simple bonding considerations would lead to a ${ }^{2} \Pi$ ground state, since one would therefore expect the $\mathrm{Si} p \sigma$ electron to pair with the $4 s \sigma \mathrm{Cu}$ or $5 s \sigma \mathrm{Ag}$ electron, leaving a lone $\mathrm{Si} p \pi$ electron. The fact that this is not evidenced in either our $\mathrm{CuSi}$ or $\mathrm{AgSi}$ data raises important questions about the nature of bonding in coinage metal silicides. Although it is possible that our absorption spectra are due to transitions out of molecular excited states, this possibility is very unlikely since the absorption band intensities are highest under source conditions which typically favor cold cluster production. A more plausible explanation is that this first-order intuitive bonding picture does not adequately describe the bonding between the metal and silicon atoms. This possibility underscores the need for high level $a b$ initio studies of these systems. Yet another explanation would be that the higher atomic asymptotes are responsible for the ground states of $\mathrm{AgSi}$ and $\mathrm{CuSi}$. This point, again, would be best addressed by theoretical studies, although such a conclusion would not be in qualitative agreement with the room temperature formation of copper silicides witnessed in solid state experiments.

\section{RESULTS AND DISCUSSION: CRLAS OF AgSi: THE $B-X\left({ }^{2} \Sigma-{ }^{2} \Sigma\right)$ AND $C-X\left({ }^{2} \Sigma-{ }^{2} \Sigma\right)$ ROVIBRONIC SYSTEMS}

In this section, the rovibronic spectra of the AgSi molecule are presented. A total of 20 rovibronic bands occurring between 365 and $385 \mathrm{~nm}$ have been measured and analyzed to yield spectroscopic constants for the $X, B$, and $C^{2} \Sigma$ states of AgSi. We have adopted the $B$ and $C$ state designations for the upper states based on the fact that the observed transitions are too high in energy to correspond to the first excited state. Rotational analyses together with the measured vibronic isotope splittings uniquely determine both the spectral carrier and vibronic assignment. From the constants derived in this work, Morse potentials have been constructed and a Franck-Condon analysis has been carried out to account for the relative band intensities of the two vibronic systems.

CRLAS spectra were obtained with a Lambda fl3002e dye laser operating on PBBO laser dye mixed in $p$-dioxane and consist of low $\left(0.3 \mathrm{~cm}^{-1}\right)$ and high $\left(0.04 \mathrm{~cm}^{-1}\right)$ resolution scans. The spectra were calibrated using the known spectra of the $C_{3}$ cluster radical, ${ }^{23}$ which is readily produced in our plasma reactor source either by seeding the helium carrier gas with methane or bubbling through a variety of organic solvents. The data are then corrected (in wavelength) with a first-order polynomial expression. Of the 20 bands measured, 5 have been rotationally fit and more than a dozen have exhibited well-resolved vibronic isotope shifts. Accurate determination of the isotope shifts is possible only in the 


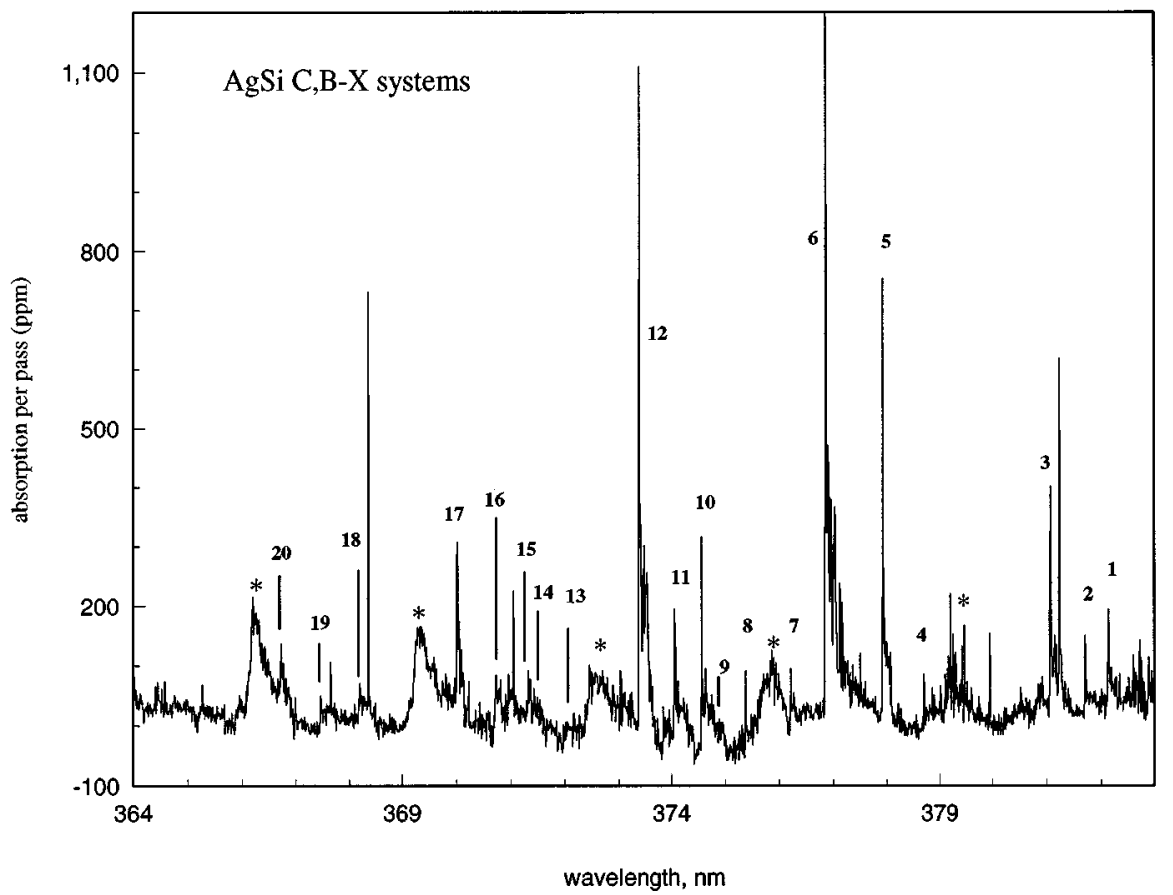

FIG. 3. Low resolution $\left(0.3 \mathrm{~cm}^{-1}\right)$ survey scan for the $B-X$ and $C-X$ systems of AgSi. Twenty vibronic bandhead positions are numbered, with their respective assignments given in Table I. The asterisks denote bands of the $H-X$ system of $\mathrm{Si}_{2}$.

high resolution scans and is found to be consistent with the vibronic assignment. Low resolution isotope shifts are in qualitative agreement with the predicted values. In addition to the upper state information obtained in this work, the observation of hot band transitions has allowed several ground state constants to be accurately determined.

\section{A. Vibrational analysis and low resolution data}

Low resolution survey scan data for the $B-X$ and $C-X$ systems of $\mathrm{AgSi}$ are presented in Fig. 3. Vibrational energy levels (of the ${ }^{107} \mathrm{Ag}^{28} \mathrm{Si}$ isotope) for the respective states were derived from bandhead positions and were fit to the expression

$$
\nu=\nu_{0}+\omega_{\epsilon}(v+1 / 2)+\omega_{\epsilon} \chi_{\epsilon}(v+1 / 2)^{2} .
$$

First-order anharmonicity was found to be sufficient for a satisfactory fit. The results of the vibronic analysis are given in Table I, with the assignments as indicated. Initial attempts to fit the $B$ state as a possible spin-orbit manifold of the ground electronic state proved unsuccessful, making it necessary to invoke a second upper electronic state in order to account for the observed bandhead positions. In the majority of the bands, only the heads are well resolved, precluding a rotational analyses for each rovibronic manifold. For this reason, vibronic origins obtained in the rotational analyses are not used in the vibrational analysis. This fact, however, does not introduce significant error to the fitted vibrational frequencies since the origin-to-bandhead distance determined in the rotationally analyzed bands is nearly constant. From the vibrational analysis, the ground and excited state well depths have been estimated (assuming Morse oscillators), using the expression

$$
D^{e}=\omega_{\epsilon}^{2} / 4 \omega_{\epsilon} \chi_{\epsilon} .
$$

The relative intensities of the $0-0,1-0,1-1$, and $0-1$ bands exhibit an interesting pattern. For transitions out of the ground vibronic level, $\Delta \nu=0$ transitions are favored, whereas for transitions out of the excited vibrational levels of the ground state, $\Delta \nu=1$ transitions are favored. This effect must be a result of the associated Franck-Condon factors, since population arguments alone would lead to 1-1 transitions which should be of greater or equal intensity to the $0-1$ bands. From the FC analysis presented later, this is indeed found to be the case. Verification of the vibronic assignment

TABLE I. AgSi vibronic bandhead positions and assignments.

\begin{tabular}{cccc}
\hline \hline Number & Frequency & $B-X\left(\nu^{\prime}-\nu^{\prime \prime}\right)$ & $C-X\left(\nu^{\prime}-\nu^{\prime \prime}\right)$ \\
\hline 1 & 26172.0 & $0-1$ & \\
2 & 26202.0 & & $1-2$ \\
3 & 26245.2 & & $0-1$ \\
4 & 26409.6 & $1-1$ & \\
5 & 26463.6 & $0-0$ & $0-0$ \\
6 & 26539.1 & & \\
7 & 26585.3 & $3-2$ & $3-2$ \\
8 & 26644.3 & $2-1$ & \\
9 & 26687.8 & & $2-1$ \\
10 & 26701.5 & $1-0$ & $1-0$ \\
11 & 26737.6 & & $4-2$ \\
12 & 26786.7 & & \\
13 & 26874.7 & $3-1$ & $3-1$ \\
14 & 26927.3 & & $2-0$ \\
15 & 26936.4 & $2-0$ & $5-2$ \\
16 & 26979.8 & & $3-1$ \\
17 & 27031.2 & & $3-0$ \\
18 & 27164.2 & & \\
19 & 27219.3 & & \\
20 & 27274.2 & & \\
\hline \hline
\end{tabular}


TABLE II. Predicted vibronic isotope shifts $\left(\right.$ in $\mathrm{cm}^{-1}$ ) based on fitted vibrational frequencies for the $B-X$ and $C-X$ systems of ${ }^{107,109} \mathrm{Ag}^{28} \mathrm{Si}$ isotopes.

\begin{tabular}{ccc}
\hline \hline $\mathrm{v}^{\prime}-\mathrm{v}^{\prime \prime}$ & $B-X$ & $C-X$ \\
\hline $0-0$ & -0.05147 & -0.04265 \\
$1-0$ & 0.39543 & 0.42757 \\
$2-0$ & 0.82823 & 0.89131 \\
$3-0$ & 1.2470 & 1.3486 \\
$0-1$ & -0.60702 & -0.5982 \\
$1-1$ & -0.16012 & -0.12795 \\
$2-1$ & 0.27268 & 0.33579 \\
$3-1$ & 0.69138 & 0.79305 \\
$0-2$ & -1.1569 & -1.14721 \\
$1-2$ & -0.70919 & -0.67699 \\
$2-2$ & -0.27639 & -0.21325 \\
$3-2$ & 0.1423 & 0.24401 \\
$0-3$ & -1.6987 & -1.6898 \\
$1-3$ & -1.2518 & -1.2195 \\
$2-3$ & -0.81898 & -0.75581 \\
$3-3$ & -0.40028 & -0.29855 \\
\hline \hline
\end{tabular}

given above is facilitated by comparison of the predicted and measured isotope shifts.

\section{B. Vibronic isotope shifts}

Derivation of the expressions for the isotope shifts can be found in Ref. 25 and only the relevant results will be presented here. Comparison of predicted isotope splittings based on measured molecular constants and $\rho$, the square root of the reduced mass ratio, allows a direct test of a given vibronic assignment. Using the values $\omega_{\epsilon}$ and $\omega_{\epsilon} \chi_{\epsilon}$ obtained from the upper and lower state vibrational fits, the isotope shifts can be predicted for the given vibronic assignment. In the case of $\mathrm{AgSi}$, the two dominant molecular isotopes are obtained by combining the two nearly equally abundant isotopes of the $\mathrm{Ag}$ atom $\left({ }^{107} \mathrm{Ag}:{ }^{109} \mathrm{Ag}=52: 48\right)$ with the single most abundant of the $\mathrm{Si}$ atom $\left({ }^{28} \mathrm{Si}:{ }^{29} \mathrm{Si}:{ }^{30} \mathrm{Si}=92: 5: 3\right)$. In Table II, the predicted isotope shifts are given as a function of upper and lower electronic state vibrational quanta, for the $B-X$ and $C-X$ systems. From the values listed in Table II, it is seen that the isotope shift will be negative for many of the bands. This causes the bandheads of the respective isotopes to effectively "switch positions" in, for instance, the $0-1$ hot band. This fact is reflected in the rotational fits which follow. These predicted isotope shifts are compared with the measured values in Table III, obtained primarily in high resolution $\left(0.04 \mathrm{~cm}^{-1}\right)$ etalon scans, and show the excellent agree-

TABLE III. Predicted vs measured isotope shifts for the $B-X$ and $C-X$ rovibronic band systems of $\mathrm{AgSi}$.

\begin{tabular}{rccrcr}
\hline \hline & \multicolumn{2}{c}{$B-X$} & & \multicolumn{2}{c}{$C-X$} \\
\cline { 2 - 3 } \cline { 5 - 6 } $\mathrm{v}^{\prime}-\mathrm{v}^{\prime \prime}$ & Predicted & Measured & & Predicted & Measured \\
\hline $1-0$ & 0.395 & $0.380(20)$ & & 0.427 & $0.433(20)$ \\
$2-0$ & 0.828 & $0.80(15)^{\mathrm{a}}$ & & 0.891 & $0.905(20)$ \\
$3-0$ & 1.247 & $\cdots$ & & 1.348 & $1.20(15)^{\mathrm{a}}$ \\
$0-1$ & -0.607 & $-0.575(20)$ & & -0.598 & $-0.557(20)$ \\
\hline \hline
\end{tabular}

${ }^{\mathrm{a}}$ Values taken from low resolution (grating scan) data.

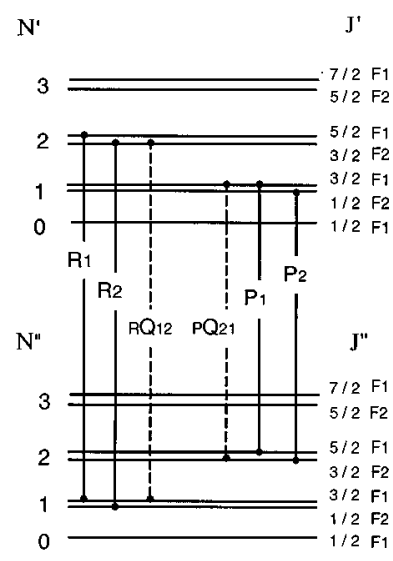

FIG. 4. Detail of the six possible rotational branches of a ${ }^{2} \Sigma-{ }^{2} \Sigma$ band. The signature of such a system is a splitting of each rotational line which increases linearly with $N$, the rotational quantum number. Because the splitting is not resolved in these scans, the bands fit equally well to a ${ }^{1} \Sigma-{ }^{1} \Sigma$ system.

ment between the two (to within a fraction of the probe laser bandwidth). These data are quantitatively consistent with the given vibronic assignment.

\section{Rotational analysis}

Of the 20 vibronic bands measured, 5 have been rotationally analyzed, including the $0-1 C-X$ band of the ${ }^{109} \mathrm{Ag}^{28} \mathrm{Si}$ isotope. From molecular orbital considerations discussed earlier, combined with the fact that the observed spectra exhibited no $Q$ branches, the bands were fit as ${ }^{2} \Sigma-{ }^{2} \Sigma$ transitions. Term values for the two spin-rotation states are given by the usual expressions. ${ }^{24}$ The resulting rovibronic band structure consists of six branches including two $R$ branches, two $P$ branches, and two very weak "satellite" $Q$ branches, as shown in Fig. 4. The signature of such a band system will be a splitting of each of the rotational lines into a doublet, which will increase linearly with $N$. In our case, due to the limited bandwidth of our probe laser $\left(0.04 \mathrm{~cm}^{-1}\right)$, this splitting is not expected to become resolved, except for very high $N$, where a broadening of the lines might become apparent. For this reason, in the fits that follow, the spinrotation constant $\gamma$ is set equal to zero. This leads to a band system which essentially looks the same as a ${ }^{1} \Sigma-{ }^{1} \Sigma$ system. Similarly, attempts to include the distortion constant $D_{e}$ in the fits led to either negative distortion constants or values with unacceptable uncertainties. The reason for this is seen when the Kratzer relation $\left(D_{e}=4 B_{e}^{3} / \omega_{\epsilon}^{2}\right)$ is employed to estimate the magnitude of $D_{e}$. For the $\nu^{\prime}=0$ upper electronic state level, using the experimentally determined constants, $D_{0} \cong 1.016 \times 10^{-7} \mathrm{~cm}^{-1}$, which, even for $N=20$, leads to a correction of only $\sim 0.02 \mathrm{~cm}^{-1}$, or roughly $1 / 2$ of our laser bandwidth. At $N=30$, this correction increases to 0.085 $\mathrm{cm}^{-1}$, but broadening of the lines due to the rotational isotope effect is on the same order, so it is not possible to distinguish between the two effects. In order to include distortion in the fits, we would require lines up to at least $N=40$, which would require substantially hotter rotational temperatures than those achieved in the current studies $(\sim 50$ $\mathrm{K})$. 


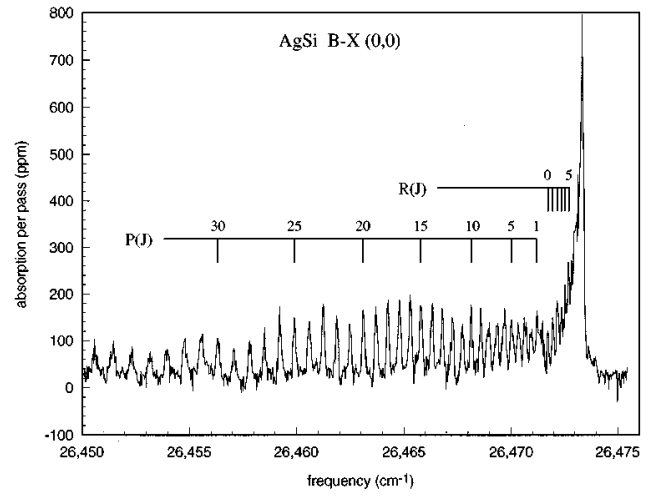

FIG. 5. Rotationally resolved CRLAS spectrum of the $0-0$ vibronic band of the $B-X$ system of $\mathrm{AgSi}$, with the assignment as indicated.

Of the 20 vibronic bands measured, the $0-0$ and $1-0$ bands of the $B-X$ and $C-X$ systems and the $0-1$ hot band of the $C-X$ system have been rotationally analyzed. The results of the fits for the two 0-0 bands are shown in Figs. 5 and 6; the associated line positions and residuals can be supplied by the authors upon request. Evident in the spectra are the isotope shifts for the various bands, which frequently lead to severely congested spectra. This tends to limit the number of lines which can be confidently used in some of the fits, which subsequently leads to higher residuals for the $\Delta \nu>0$ bands. In all fits, the residuals are found to be within the uncertainty associated with the probe laser bandwidth. For the bands which originate in the ground vibronic level, fixing the lower state constant to $0.13023 \mathrm{~cm}^{-1}$ (the average value obtained in the individual fits) substantially reduces the uncertainty of the upper state constants and decreases residuals. Results of the rotational fits for the heavier isotope of the $C-X(0-1)$ hot band are shown in Fig. 7. In the rotational isotope effect, the rotational constants for the respective isotopes scale as $\rho^{2}$. Comparison of the rotational constants of the two isotopes is found to be in agreement with the predicted quantities, as are the values of $\alpha_{e}$. All of the molecular constants derived from this work are given in Table IV. Comparison of these results with those of $\mathrm{CuSi}$ indicate important similarities in bonding which will be discussed in the following sections.

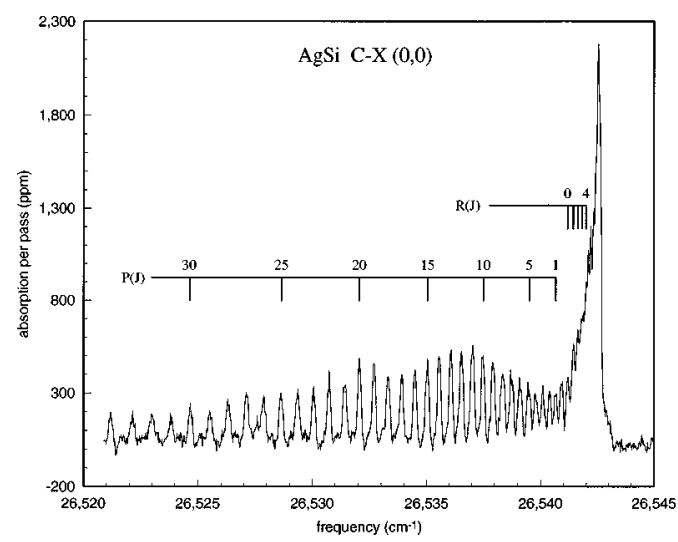

FIG. 6. Rotationally resolved CRLAS spectrum of the $0-0$ band of the $C-X$ system of $\mathrm{AgSi}$.

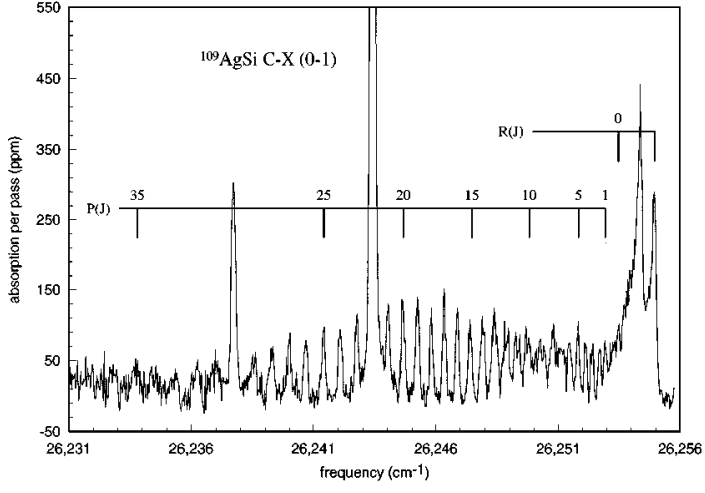

FIG. 7. Rotationally resolved spectrum of the $0-1$ hot band of the $C-X$ system of AgSi.

\section{Discussion and comparison with CuSi}

From the properties measured in this study, a quantitatively and qualitatively consistent picture of the bonding in the three electronic states is evident. The ${ }^{2} \Sigma$ ground state, which is bound by $1.88 \mathrm{eV}$, has a vibrational frequency of $296.9 \mathrm{~cm}^{-1}$ with an associated anharmonicity of $1.45 \mathrm{~cm}^{-1}$. The $B$ state, also of ${ }^{2} \Sigma$ symmetry, is bound by only $\sim 1 \mathrm{eV}$ and possesses an appropriately lower vibrational frequency of $241.9 \mathrm{~cm}^{-1}$. The $C$ state, which is bound by $1.5 \mathrm{eV}$, has a vibrational frequency of $250.2 \mathrm{~cm}^{-1}$, which, accordingly, falls between the $B$ and $X$ state values. Vibrational constants obtained indicate a $19 \%$ and $15 \%$ decrease for the $B$ and $C$ state vibrational frequencies upon electronic excitation, respectively, in qualitative agreement with the respective binding energies. Similarly, the rotational constants are in qualitative agreement with the vibrational frequencies, although the $B$ state value of $0.12176 \mathrm{~cm}^{-1}$ is slightly larger than the $C$ state value of $0.12052 \mathrm{~cm}^{-1}$. This violation of Badger's rule is most likely due to the subtle bonding differences between the two states. For the ground vibronic level, a bond length of $2.41 \AA$ is obtained, while bond lengths for the $B$ and $C$ zero-point vibronic levels are 2.50 and $2.51 \AA$, respectively. Using the constants obtained in this work, Morse potentials can be constructed with the expression

$$
V(r)=D^{e}\left[1-\exp \left(-\beta\left(r-r_{e}\right)\right]^{2},\right.
$$

where $\beta=8 \pi^{2} c \mu \omega_{\epsilon} \chi_{\epsilon} / h, D^{e}$ and $r_{e}$ are the well depth and bond length, respectively, $h$ is Planck's constant, and $\mu$ is the

TABLE IV. Molecular constants for the ${ }^{107} \mathrm{Ag}^{28} \mathrm{Si}$ molecule. All values are given in $\mathrm{cm}^{-1}$, unless otherwise indicated.

\begin{tabular}{lccc}
\hline \hline & \multicolumn{1}{c}{$X$} & \multicolumn{1}{c}{$B$} & \multicolumn{1}{c}{$C$} \\
\hline$\nu_{00}$ & \multicolumn{1}{c}{$\ldots$} & $26462.18(4)$ & $26437.45(4)$ \\
$\omega_{\epsilon}$ & 296.9 & 241.9 & 250.2 \\
$\omega_{\epsilon} \chi_{\epsilon}$ & $1.45(8)$ & $1.85(5)$ & $1.29(3)$ \\
$B_{0}$ & $0.13023(2)$ & $0.12176(5)$ & $0.12052(2)$ \\
$B_{1}$ & $0.12995(3)$ & $0.12133(10)$ & $0.12054(2)$ \\
$r_{e}, \AA$ & 2.413 & 2.496 & 2.509 \\
$\alpha_{e}$ & $2.7 \times 10^{-4}$ & $4.3 \times 10^{-4}$ & $\ldots$ \\
$D_{e} \times 10^{7 \mathrm{a}}$ & 1.02 & 1.23 & 1.12 \\
$D^{e}(\mathrm{eV})$ & 1.88 & 0.98 & 1.51 \\
\hline \hline
\end{tabular}

${ }^{a}$ Calculated using Eq. (7), with constants derived from this work. 


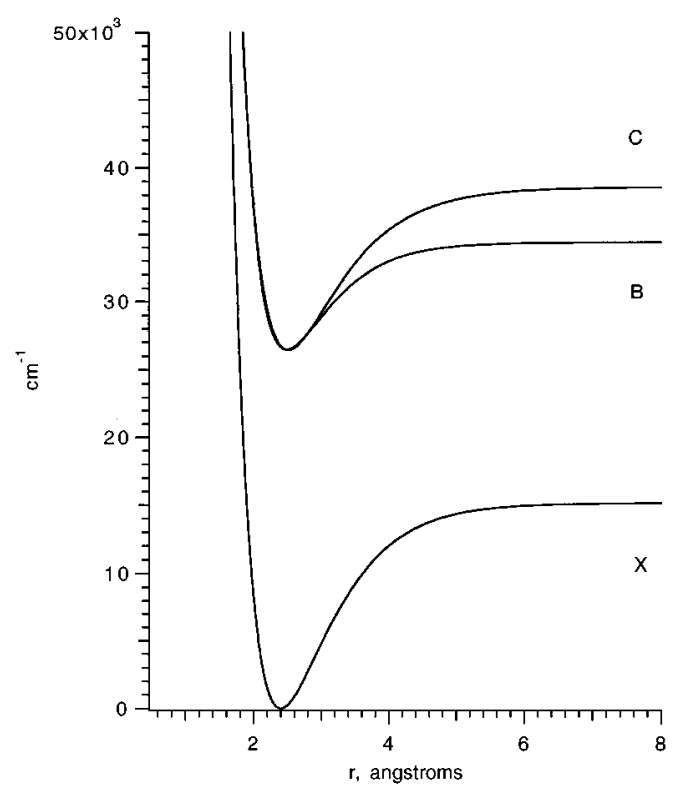

FIG. 8. Morse potentials for the $X, B$, and $C$ states of $\mathrm{AgSi}$, derived from spectroscopic constants obtained in this work.

reduced mass. The resultant qualitative potential wells are shown in Fig. 8. Using the experimentally determined molecular constants, a Franck-Condon analysis has been performed, in order to check the relative intensities of the vibronic bands within a given vibronic system. By specifying the upper and lower state vibrational frequencies, anharmonicities, and bond lengths, the appropriate vibrational wave functions are constructed and the overlap integrals are evaluated. The computer program for the following FC analysis was written and supplied to us by Dr. Don Arnold. To compare the measured intensities to those predicted in a FC analysis, a vibrational temperature must be specified. Results of the FC analysis for the $C-X$ and $B-X$ systems are shown in Fig 9(a), assuming a vibrational temperature of $300 \mathrm{~K}$. The predicted relative intensities are in excellent agreement with the experimental values shown in Fig. 9(b), indicating the consistency of the molecular properties derived from our spectral analysis. This suggests that all of the states measured in this work are at least qualitatively well described by the above potentials in the measured regions.

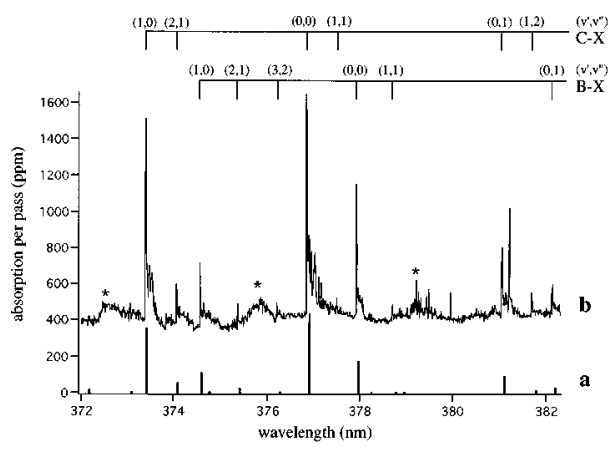

FIG. 9. Comparison of measured relative intensities for the $C-X$ and $B-X$ systems of AgSi with predicted intensities from a Franck-Condon simulation, using experimentally determined constants and assuming a vibrational temperature of $300 \mathrm{~K}$. The asterisks denote $\mathrm{Si}_{2}$ bands.
TABLE V. Ground state molecular constants for $\mathrm{AgSi}$ and $\mathrm{CuSi}$.

\begin{tabular}{lcc}
\hline \hline & $\mathrm{CuSi}$ & $\mathrm{AgSi}$ \\
\hline Symmetry & ${ }^{2} \Sigma$ & ${ }^{2} \Sigma$ \\
$r_{e}(\AA)$ & 2.28 & 2.40 \\
$\omega_{e}\left(\mathrm{~cm}^{-1}\right)$ & 320 & 297 \\
$D_{e}(\mathrm{eV})$ & $2.25^{\mathrm{a}}$ & $1.88(10)$ \\
\hline \hline
\end{tabular}

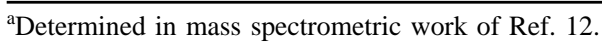

Comparison of the $\mathrm{AgSi}$ results with those presented in Ref. 13 for $\mathrm{CuSi}$ indicates interesting similarities between the two coinage metal silicides. The important molecular properties for the ground states of the two species are given in Table V. For both molecules, our data indicate ${ }^{2} \Sigma$ ground states with similar bond lengths and vibrational frequencies. In fact, if we scale the two vibrational frequencies with the square root of the ratio of the reduced masses $\left[\omega \propto(\kappa / \mu)^{1 / 2}\right]$, we find that the force constants $(\kappa)$ for the two molecules are nearly identical. Additionally, our calculated $1.88 \mathrm{eV}$ value of the well depth of $\mathrm{AgSi}$ is in excellent agreement with the $1.80 \mathrm{eV}$ mass spectrometric value obtained by Riekert et al. ${ }^{12}$ Comparison of these values with the binding energy for $\mathrm{CuSi}$ also obtained in Ref. 12 is also in agreement. This comparison of well depth, however, is somewhat limited due to the small number of ground state vibrational levels probed in our work and the associated uncertainties of assuming a Morse potential. The bond lengths of the two coinage metal silicides extracted from our rotational analyses are also similar, with the AgSi value only $0.12 \AA$ larger than that of $\mathrm{CuSi}$. These bonding trends are qualitatively understood by considering the properties of the constituent atoms which are listed in Table VI.

Comparison of the electronegativities of the individual atoms predicts that the resultant molecular bonds for both $\mathrm{AgSi}$ and $\mathrm{CuSi}$ will be almost entirely covalent, with virtually no ionic character. Therefore, the diatom bond lengths of the various combinations should be very close to the sums of the individual covalent radii. It is also interesting to note that the differences between the atomic radii and the associated covalent radii are almost the same for the different atoms. If we add the covalent radii of $\mathrm{Cu}$ and $\mathrm{Si}$, a value of $2.24 \AA$ is obtained, while for $\mathrm{AgSi}$, this sum equals $2.45 \AA$. These values are very close to the 2.28 and $2.40 \AA$ bond lengths extracted from our data. This comparison indicates the bonding trends expected for the two coinage metals silicides, with important implications for other transition metal silicide compounds. From these considerations and the experimental data, we conclude that ground state bonding between the $\mathrm{Si}$ atom and either $\mathrm{Ag}$ or $\mathrm{Cu}$ is primarily covalent, but does not

TABLE VI. Atomic properties for ground state $\mathrm{Si}, \mathrm{Cu}$, and $\mathrm{Ag}$.

\begin{tabular}{lccc}
\hline \hline & $\mathrm{Si}$ & $\mathrm{Cu}$ & $\mathrm{Ag}$ \\
\hline${\text { Atomic radius }(\AA)^{\mathrm{a}}}^{\mathrm{a}}$ & 1.46 & 1.57 & 1.75 \\
Dimer radius/2 $(\AA)^{\mathrm{b}}$ & 1.12 & 1.12 & 1.33 \\
Electronegativity & 1.90 & 1.90 & 1.93 \\
\hline \hline
\end{tabular}

${ }^{\mathrm{a}}$ Quantum mechanical value.

${ }^{\mathrm{b}}$ Experimental values (covalent radius). 
arise from a simple valence bond treatment, since this would most likely result in ${ }^{2} \Pi$ ground states, as discussed earlier.

For both $\mathrm{Cu}$ and $\mathrm{Ag}$ silicides, the excited states are considerably less bound than the ground state, suggesting the excitation is possibly of $\sigma-\sigma^{*}$ character. Excited state asymptotic energies for the AgSi molecule can be calculated, since both upper and lower well depths have been determined. Using the relation

$$
\Delta E=\nu_{e}+D^{e^{\prime}}-D^{e^{\prime \prime}}
$$

we arrive at an upper state atomic excitation energy of $\sim 23400$ and $19200 \mathrm{~cm}^{-1}$, for the $C$ and $B$ states, respectively. These values are within the range for either excited metal or silicon atom atomic asymptotes, assuming that ground state atoms combine to form the ground state molecule. Rigorously, precise determination of the respective well depths would be required for accurate asymptotic limits, and this is not possible in the current case since our analysis is based on only a few of the lower vibrational states in each electronic manifold. In light of this, the potentials presented here are appropriate only for qualitative purposes. Ab initio studies of both the ground and excited states of AgSi would be of considerable value in rationalizing the trends observed here.

\section{SUMMARY}

In this paper, we have reported the first spectral characterization of the AgSi molecule, which is generated in a pulsed UV laser plasma reactor. The rovibronic absorption spectra of the ${ }^{2} \Sigma X, B$, and $C$ states have been obtained with the CRLAS method, and a time-of-flight mass spectrometer has been used to obtain the first mass spectra of polyatomic silver silicide clusters. The mass spectra obtained with different ionization wavelengths suggests that photofragmentation does not severely distort the measured cluster distributions. From the direct absorption data, we conclude that bonding in both silver and copper silicides is primarily covalent in nature with appropriately similar bond lengths and force constants. Our results also suggest the facile formation of coinage metal silicides from ground state atoms, consistent with the low temperature formation of metal silicides witnessed in solid state studies. Extension of these studies to include other metal silicide species is currently in progress in our laboratory.

\section{ACKNOWLEDGMENTS}

This research was supported by the Air Force Office of Scientific Research under Grant No. F49620-93-1-0278. J.J.S. thanks I.B.M. for a predoctoral fellowship during part of this research.

${ }^{1}$ See, e.g., K. K. Ng, Properties of Silicon (EMIS Data Reviews Series No. 4, London, UK, 1988), pp. 799-826.

${ }^{2}$ See, for example, M. Setton, J. Van der Spiegel, and B. Rothman, Appl. Phys. Lett. 57, 357 (1990).

${ }^{3}$ See, for example, S. Hymes, S. P. Muraka, C. Shepard, and W. A. Langford, J. Appl. Phys. 71, 4623 (1992).

${ }^{4}$ X. Xide and Z. Kaiming, Progr. Surf. Sci. 28, 71 (1988).

${ }^{5}$ U. Kambli, M. von Allmen, N. Saunders, and A. P. Miodownik, Appl. Phys. A 36, 189 (1985).

${ }^{6}$ S. Hassam, M. Gaune-Escard, and J. P. Bros, Ber. Bunsenges Phys. Chem. 87, 785 (1983).

${ }^{7}$ G. Le Lay, J. Vac. Sci. Technol. B 1, 354 (1983)

${ }^{8}$ M. Arnold, J. Fruhauf, and H. G. Schneider, Cryst. Res. Technol. 18, 1015 (1983)

${ }^{9}$ F. Rollert, N. A. Stolwijk, and H. Mehrer, J. Phys. D 20, 1148 (1987).

${ }^{10}$ H. Tokutaka, K. Nishimori, S. Nomura, A. Tanaka, and K. Takashima, Surf. Sci. 115, 79 (1982)

${ }^{11}$ J. Budinavicius, L. Pranevicius, and S. Tamulevicius, Phys. Status Solidi A 114, K25 (1989)

${ }^{12}$ G. Riekert, P. Lamparter, and S. Steeb, Z. Metall. 72, 765 (1981).

${ }^{13}$ J. J. Scherer, J. B. Paul, C. P. Collier, and R. J. Saykally, J. Chem Phys. 102, 5190 (1995).

${ }^{14}$ A. O'Keefe and D. A. G. Deacon, Rev. Sci. Instrum. 59, 2544 (1988); see also A. J. Ramponi, Fred P. Milanovich, T. Kan, and D. Deacon, Appl. Opt. 27, 4606 (1988); J. M. Herbelin, J. A. McKay, M. A. Kwok, R. H. Ueunten, D. S. Urevig, D. J. Spencer, and D. J. Benard, ibid. 19, 144 (1980); J. M. Herbelin and J. A. McKay, ibid. 20, 3341 (1980); D. Z. Anderson, J. C. Frisch, and C. S. Masser, ibid. 23, 1238 (1984).

${ }^{15}$ A. O'Keefe, J. J. Scherer, A. L. Cooksy, R. Sheeks, J. Heath, and R. J. Saykally, Chem. Phys. Lett. 172, 214 (1990).

${ }^{16}$ D. Romanini and K. K. Lehmann, J. Chem. Phys. 99, 6287 (1993).

${ }^{17}$ T. Yu and M. C. Lin, J. Am. Chem. Soc. 115, 4371 (1993).

${ }^{18}$ G. Meijer, M. G. H. Boogaarts, R. T. Jongma, D. H. Parker, and A. M. Wodtke, Chem. Phys. Lett. 217, 112 (1994).

${ }^{19}$ P. Zalicki, Y. Ma, R. N. Zare, E. H. Wahl, J. R. Dadamio, T. G. Owano, and C. H. Kruger, Chem. Phys. Lett. (submitted).

${ }^{20}$ B. Simard, P. A. Hackett, A. M. James, and P. R. R. Langridge-Smith, Chem. Phys. Lett. 186, 415 (1991).

${ }^{21}$ G. A. Bishea and M. D. Morse, J. Chem. Phys. 95, 5646 (1991).

${ }^{22}$ G. A. Bishea, N. Marak, and M. D. Morse, J. Chem. Phys. 95, 5618 (1991).

${ }^{23}$ L. Gausset, G. Herzberg, A. Lagerqvist, and B. Rosen, Astrophys. J. 142, 45 (1965).

${ }^{24}$ G. Herzberg, Spectra of Diatomic Molecules (Van Nostrand, New York, 1953), Chap. VI 